\title{
Optimal combination chemotherapy and chemoradiotherapy with etoposide for advanced cervical squamous cancer cells in vitro
}

\author{
TETSUJI TANAKA ${ }^{1}$, TAO BAI ${ }^{1}$, KAZUNORI YUKAWA $^{2}$ and NAOHIKO UMESAKI ${ }^{1}$ \\ Departments of ${ }^{1}$ Obstetrics and Gynecology and ${ }^{2}$ Physiology, Wakayama Medical University, \\ 811-1 Kimi-idera, Wakayama 641-0012, Japan
}

Received September 6, 2005; Accepted November 14, 2005

\begin{abstract}
Patients with unresectable advanced carcinoma of the uterine cervix are usually treated with chemotherapy or chemoradiotherapy. In the present study, the optimal administration protocol for etoposide in chemotherapy and chemoradiotherapy for advanced cervical cancer patients was investigated in vitro using the radio-sensitive and anticancer drug-sensitive human cervical squamous cell carcinoma cell line ME180. Therapeutic doses of concurrent irradiation reduced the cellular etoposide sensitivity in a dose-dependent manner, while postirradiation-surviving subclones established from repeatedly irradiated ME180 cells showed significantly higher etoposide sensitivities than the non-irradiated parent cells. Of the 6 monoclonal etoposide-resistant subclones established from ME180 cells, 5 were significantly radioresistant. Although the etoposide-resistant subclones were also significantly resistant to other anticancer drugs, such as cisplatin, carboplatin, nedaplatin, pirarubicin, paclitaxel and docetaxel, they were more sensitive to 5-fluorouracil, mitomycin C and SN38 than the parent cells. Flow cytometric analyses revealed that the etoposide-resistant subclones showed significantly increased cell surface expression of CD40 compared to the parent cells, which expressed undetectable levels of CD40. However, the expression of some integrin receptor subunits, such as CD29, CD49a and CD49f, was apparently reduced in the etoposide-resistant subclones. These results indicate that etoposide should be administered to advanced cervical squamous cancer patients after the completion of radiotherapy, rather than as a concurrent chemoradiotherapy. In order to kill surviving etoposideresistant cancer cells more effectively, 5-fluorouracil, mitomycin $\mathrm{C}$ and irinotecan may be candidate combination drugs for use with etoposide. Differential expression of
\end{abstract}

Correspondence to: Dr Tetsuji Tanaka, Department of Obstetrics and Gynecology, Wakayama Medical University, 811-1 Kimi-idera, Wakayama 641-0012, Japan

E-mail: tetanaka@wakayama-med.ac.jp

Key words: etoposide, drug resistance, radioresistance, squamous cell carcinoma, cervical cancer, CD40, chemoradiotherapy integrin receptors and CD40 may be involved in the acquisition of etoposide resistance by cervical squamous cancer cells.

\section{Introduction}

Patients with unresectable advanced cervical cancer are usually treated with radiotherapy against the primary lesion and metastatic lesions in pelvic and para-aortic lymph nodes, although radiotherapy alone results in a low long-term survival of these patients. There are two possible reasons why advanced cervical cancer patients show a low survival ratio after radiotherapy alone. The first is that micrometastatic lesions outside the irradiated areas may grow after radiotherapy has started, and the second is that cancer cells within the irradiated areas may not be completely killed by the primary radiotherapy alone. To improve the low survival ratio of radiotherapy alone, concurrent chemoradiotherapy has often been applied to patients with advanced cervical cancers. Although cervical squamous cell carcinoma (SCC) is considered to be highly radio-sensitive, many studies have reported that concurrent chemoradiotherapy produces better survival ratios than radiotherapy alone (1-8). In these reports, cisplatin (CDDP), mitomycin (MMC) or 5-fluorouracil (5-FU) was used in concurrent chemoradiotherapy. Furthermore, several clinical trials of concurrent chemoradiotherapy for cervical cancer patients involving CDDP, 5-FU and/or irinotecan $\mathrm{HCl}$ (CPT-11) have been performed in Japan.

Etoposide (VP16), a topoisomerase II inhibitor, is an anticancer drug used to treat various types of malignant tumors. In Japan, etoposide has been clinically used for several gynecological malignant tumors, such as choriocarcinoma, endometrial adenocarcinoma $(9,10)$ and cervical adenocarcinoma (11). Oral etoposide has been used to treat advanced cervical SCC patients, usually as a chemotherapeutic drug in tumor dormancy therapy. However, the optimal conditions for oral etoposide administration, as well as which anticancer drugs can be combined with oral etoposide for unresectable cervical cancer patients treated by radiotherapy, are currently unknown. Therefore, the aim of the present study was to investigate the optimal administration protocols of etoposide to improve the therapeutic results for unresectable advanced cervical SCC patients.

The anticancer effects of chemotherapy after radiotherapy differ markedly from those of radiotherapy after chemotherapy. For example, Tabata et al reported that primary bleomycin, 


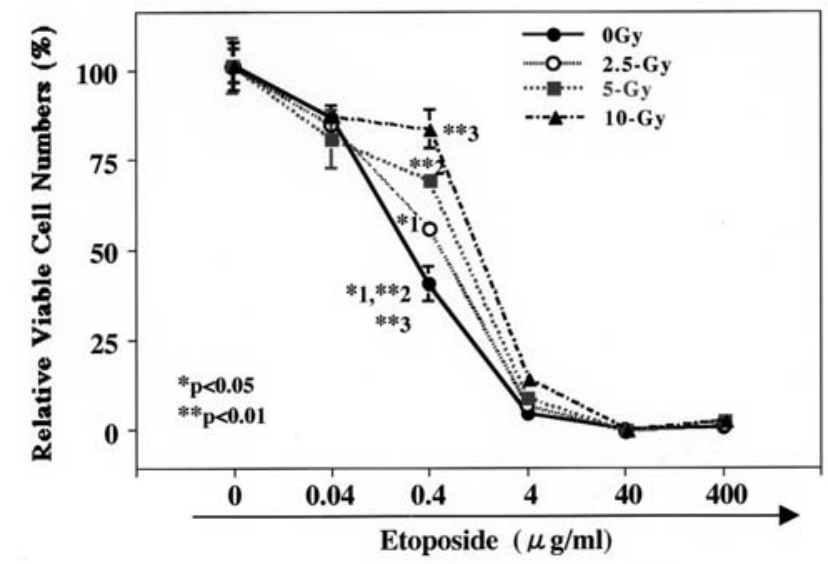

Figure 1. Effects of irradiation on the etoposide sensitivity of ME180 cells. Following the addition of various concentrations of etoposide, ME180 cells were irradiated with various doses of $\gamma$-rays within $20 \mathrm{~min}$. The solid line with closed circles shows the control etoposide sensitivity curve of cells cultured without irradiation. Dotted lines with open circles (2.5 Gy), closed squares $(5.0 \mathrm{~Gy})$ and closed triangles $(10.0 \mathrm{~Gy})$ are the etoposide sensitivity curves of irradiated cells. As shown in the data for $0.4 \mu \mathrm{g} / \mathrm{ml}$ of etoposide, irradiation significantly reduces the etoposide sensitivity in a dose-dependent manner. The cell viabilities $(\%)$ were compared between cells with and without irradiation. ${ }^{*} 1, \mathrm{p}<0.05 ;{ }^{* *} 2$ and ${ }^{* * *} 3, \mathrm{p}<0.01$.

vincristine, MMC and CDDP (BOMP) chemotherapy followed by radiotherapy had a worse survival ratio in cervical cancer patients than radiotherapy alone (12). Our previous studies revealed that the optimal administration protocols of different anticancer drugs depend on the drugs themselves in cases of combined chemoradiotherapy. For example, pirarubicin (THP) treatment before radiotherapy may have better antitumor effects on cervical SCC cells than concurrent chemoradiotherapy with THP (13), while MMC (14) and CDDP (15) should be administered concurrently with radiotherapy and/or after the completion of radiotherapy. CPT-11 should be administered after radiotherapy because SN38, an active metabolite of CPT-11, did not show a synergistic effect on cervical SCC cells in concurrent chemoradiotherapy in vitro (16). Therefore, in the present study, we initially examined when etoposide should be combined with radiotherapy using cultured SCC cells in vitro. Subsequently, to identify candidate drugs that can be administered with etoposide, we investigated the antitumor effects of different anticancer drugs on etoposideresistant cells.

CD40 is a TNF receptor family member that transduces antiapoptotic signaling and differs from apoptotic receptors, such as the TNF receptor or Fas antigen (17). Previous studies have demonstrated that the CD40-CD40 ligand (CD40L) system may be involved in the acquisition of resistance to anticancer drugs, such as etoposide, paclitaxel and doxorubicin (18-23). In our previous study, we found that CD40 expression was increased in cyclophosphamide (CPA)-resistant subclones established from the human cervical SCC cell line ME180 compared to ME180 parent cells, which expressed undetectable levels of CD40 (24). These CPA-resistant subclones showed multidrug resistance against 8 anticancer drugs, including etoposide, paclitaxel and doxorubicin. However, it is unknown as to whether the increased CD40 expression in the CPAresistant subclones is associated with the acquired multidrug resistance against etoposide, paclitaxel and doxorubicin.

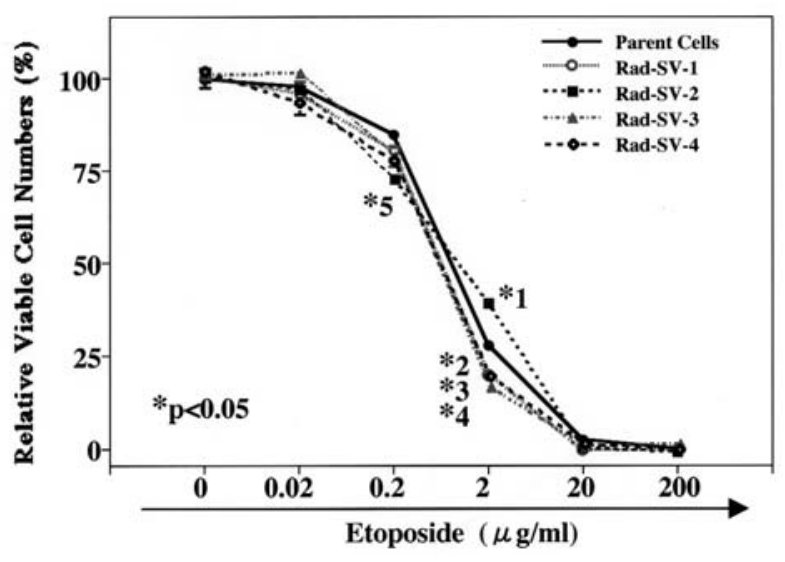

Figure 2. Etoposide sensitivities of postirradiation-surviving subclones. The etoposide-sensitivities of 4 subclones (Rad-SV-1-4) established from cells that survived repeated irradiations were compared with the etoposide sensitivity of non-irradiated ME180 cells. The solid line with closed circles shows the control etoposide sensitivity curve of the parent cells. The four dotted lines show the etoposide sensitivity curves of Rad-SV-1-4. The mean viable cell numbers without etoposide treatment were set as $100 \%$. The relative viable cell numbers after treatment with etoposide were compared between the parent cells and postirradiation-surviving subclones. Of the 4 post-irradiation surviving subclones, 3 (Rad-SV-1, Rad-SV-3 and Rad-SV-4) showed significantly higher sensitivities to etoposide than the parent cells. ${ }^{*} 1-{ }^{*} 5, \mathrm{p}<0.05$.

Moreover, the CPA-resistant subclones were also radioresistant, indicating that increased CD40 expression may be involved in the acquired radioresistance of these cells. Therefore, we also examined the relationships between increased CD40 expression and acquired radioresistance or multidrug resistance in the current study by characterizing etoposide-resistant subclones established from the ME180 human cervical cancer cell line.

\section{Materials and methods}

Cell line and cell culture. The human cervical SCC cell line ME180 (25), which is radio-sensitive (26), was obtained from the Japan Resources of Cell Bank (JRCB, Tokyo, Japan). All cells used in this study were cultured in Opti-MEM (GibcoBRL, Gaithersburg, MD) supplemented with 5\% fetal calf serum (FCS; Equitech Bio Inc., Ingram, TX), $100 \mathrm{U} / \mathrm{ml}$ penicillin (Gibco-BRL) and $100 \mu \mathrm{g} / \mathrm{ml}$ streptomycin (GibcoBRL). VP16, CDDP and THP were provided by NipponKayaku Co. Ltd. (Tokyo, Japan). Carboplatin (CBDCA) and paclitaxel were provided by Bristol-Myers Squib Japan Co. Ltd. (Tokyo, Japan). 5-FU and MMC were provided by Kyowa-Hakko Co. (Tokyo, Japan). Nedaplatin was obtained from Shionogi and Co. Ltd. (Osaka, Japan). Docetaxel was provided by Aventis Japan (Tokyo). SN38, a major active metabolite of CPT-11, was provided by Yakult Co. (Tokyo, Japan).

Radiosensitivity assay. The radiosensitivity of cultured cells was assayed using a non-RI colorimetric assay XTT kit (Boehringer-Mannheim, Mannheim, Germany). The growthinhibitory effects of radiation and etoposide on the cells were assayed as follows. Cells in the log phase were detached with $0.25 \%$ trypsin/1 mM EDTA, then cultured overnight in 96 -well plates $\left(5 \times 10^{3}\right.$ cells/well $)$. On day 2 , the cells were 


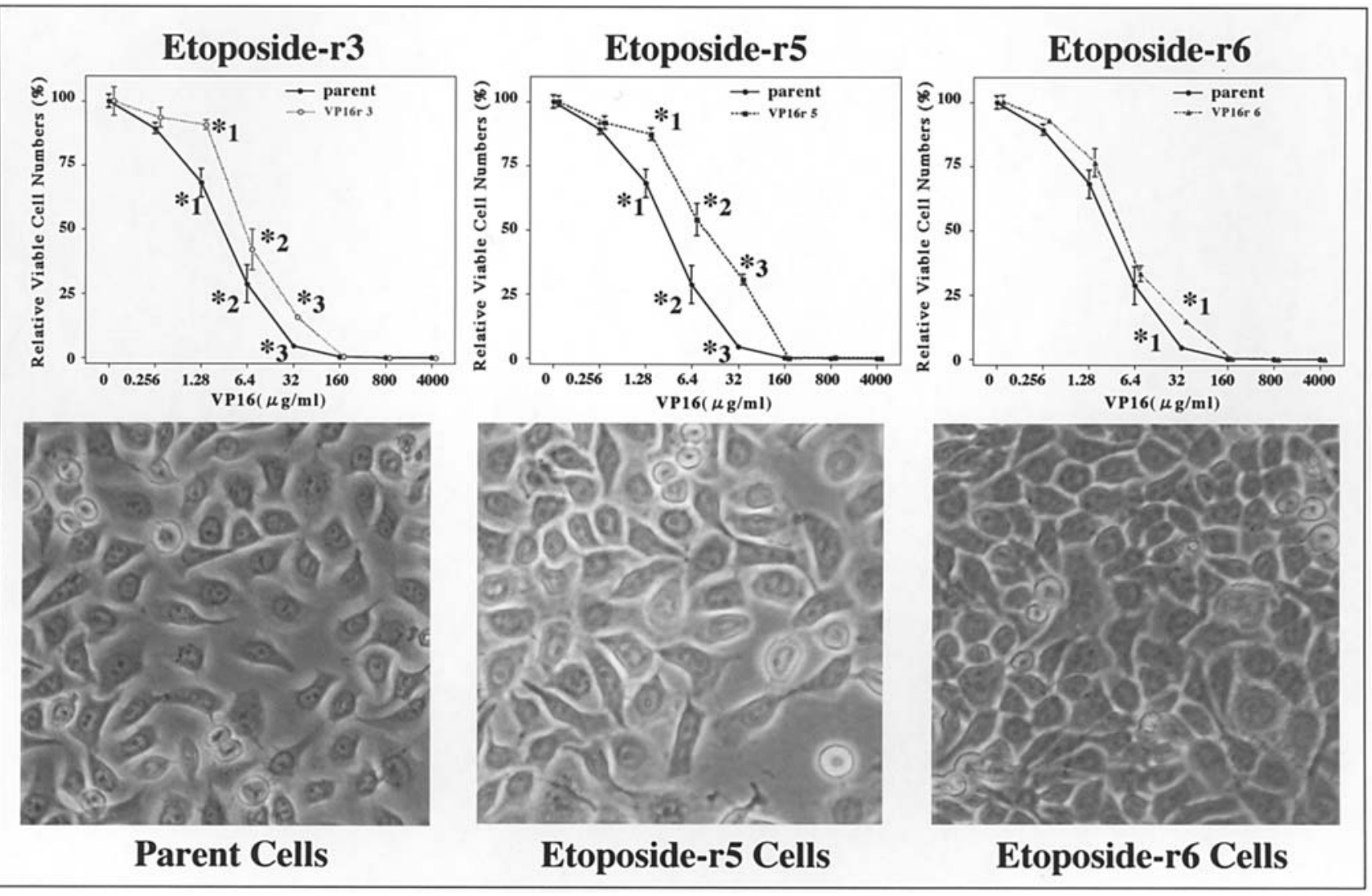

Figure 3. Establishment of etoposide-resistant subclones derived from ME180 cells. The etoposide sensitivity curves of 3 single-cell-derived etoposideresistant subclones (Etoposide-r3, Etoposide-5r and Etoposide-r6) are shown. The solid lines with closed circles show the etoposide sensitivity curves of the parent cells. Dotted lines with open circles are the etoposide sensitivity curves of the etoposide-resistant subclones. The photos in the bottom panels show the microscopic appearances of the parent cells and etoposide-resistant subclones after in vitro culture. ${ }^{*} 1-3, \mathrm{p}<0.05$.

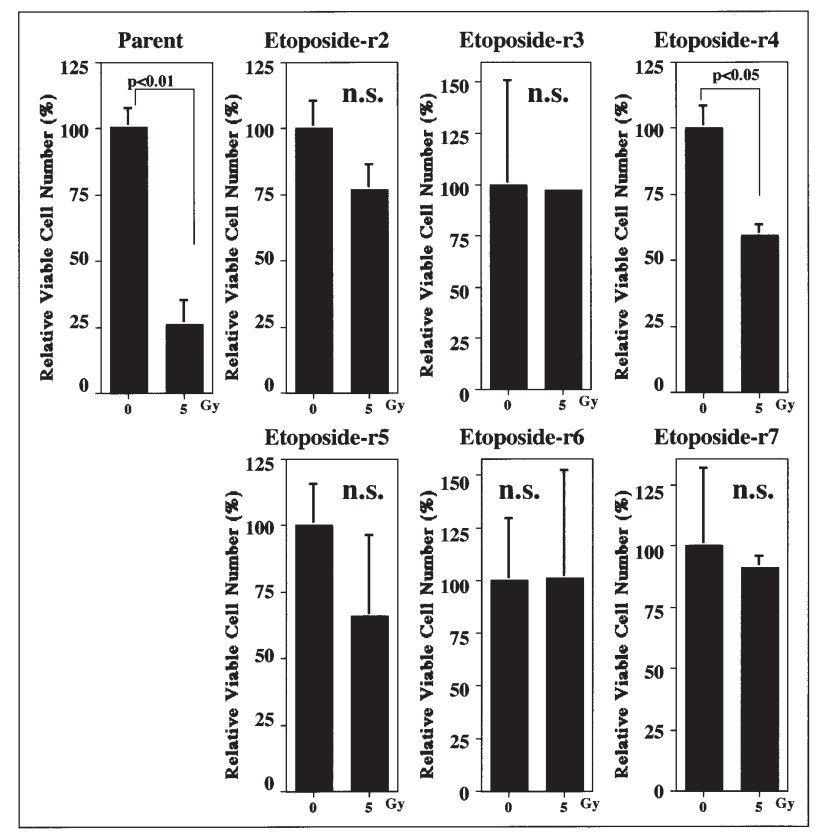

Figure 4. Radiosensitivity assays of the etoposide-resistant subclones. Radiosensitivity assays were performed on all 6 etoposide-resistant subclones (Etoposide-r2, Etoposide-r3, Etoposide-r4, Etoposide-r5, Etoposide-r6 and Etoposide-r7). The mean viable cell numbers without irradiation were set as $100 \%$. The relative viable cell numbers $(\%)$ after irradiation at 5-Gy were compared to those of the non-irradiated cells. Of the 6 etoposide-resistant subclones, 5 (Etoposide-r2, Etoposide-r3, Etoposide-r5, Etoposide-r6 and Etoposide-r7) showed significant resistance to irradiation, while the remaining subclone (Etoposide-r4) was radio-sensitive. irradiated with various doses of $\gamma$-rays using an irradiator (MBR 1520A; Hitachi-Medico, Tokyo, Japan). On day 4, the viable cells were counted using the XTT kit. To examine the modulatory effects of etoposide on the cell death induced by irradiation, cells were treated with various concentrations of etoposide and irradiated with various doses of $\gamma$-rays, followed by a 2-day culture. Finally, the relative viable cell numbers (\%) were calculated using the XTT kit. All experiments were performed 2-3 times to verify the results. Data are shown as the mean $\pm \mathrm{SD}$, and comparative data $(\mathrm{n}=6)$ were analyzed by ANOVA.

Establishment of surviving subclones after repeated irradiations. The subclones were established as follows. ME180 parent cells were cultured in five 96-well culture plates $\left(1 \times 10^{4}\right.$ cells/well) and irradiated with 10 Gy of $\gamma$-rays once a week for 4 weeks. In a preliminary experiment, more than $90 \%$ of ME180 cells were killed after a single $\gamma$-ray irradiation of $10 \mathrm{~Gy}$. Cells were collected from each of 4 wells with viable cancer cell colonies and re-cultured with a lower cell density (0.1-20.0 cells/well) in a limiting dilution study. The cloning efficiencies of the limiting dilution cultures were $<10 \%(3.7-9.1 \%)$. Finally, 4 months after the first irradiation, 4 monoclonal subclones (Rad-SV-1-4) surviving the irradiations were established.

Establishment of etoposide-resistant subclones from ME180 cells. To establish etoposide-resistant subclones, ME180 parent cells were cultured with various concentrations of 


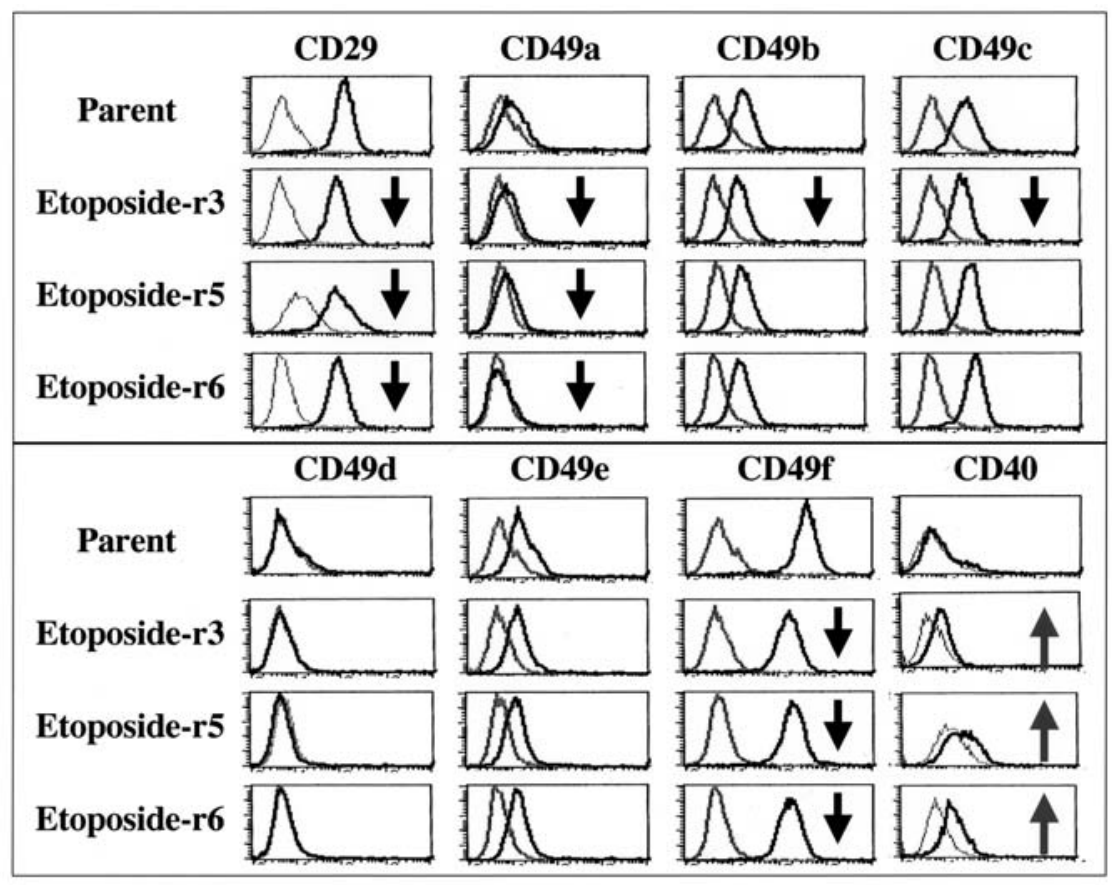

Figure 5. Comparative flow cytometric analyses of the parent cells and etoposide-resistant subclones. Flow cytometric analyses were performed simultaneously with excess doses of the antibodies to compare the expression levels between parent cells and etoposide-resistant subclones. The thin line in each figure is the negative control (secondary antibody alone). The cell surface CD40 expression is clearly increased in the etoposide-resistant subclones, while the parent cells show undetectable levels of CD40 expression. In contrast, the expression levels of CD29, CD49a and CD49f were clearly reduced in the etoposide-resistant subclones.

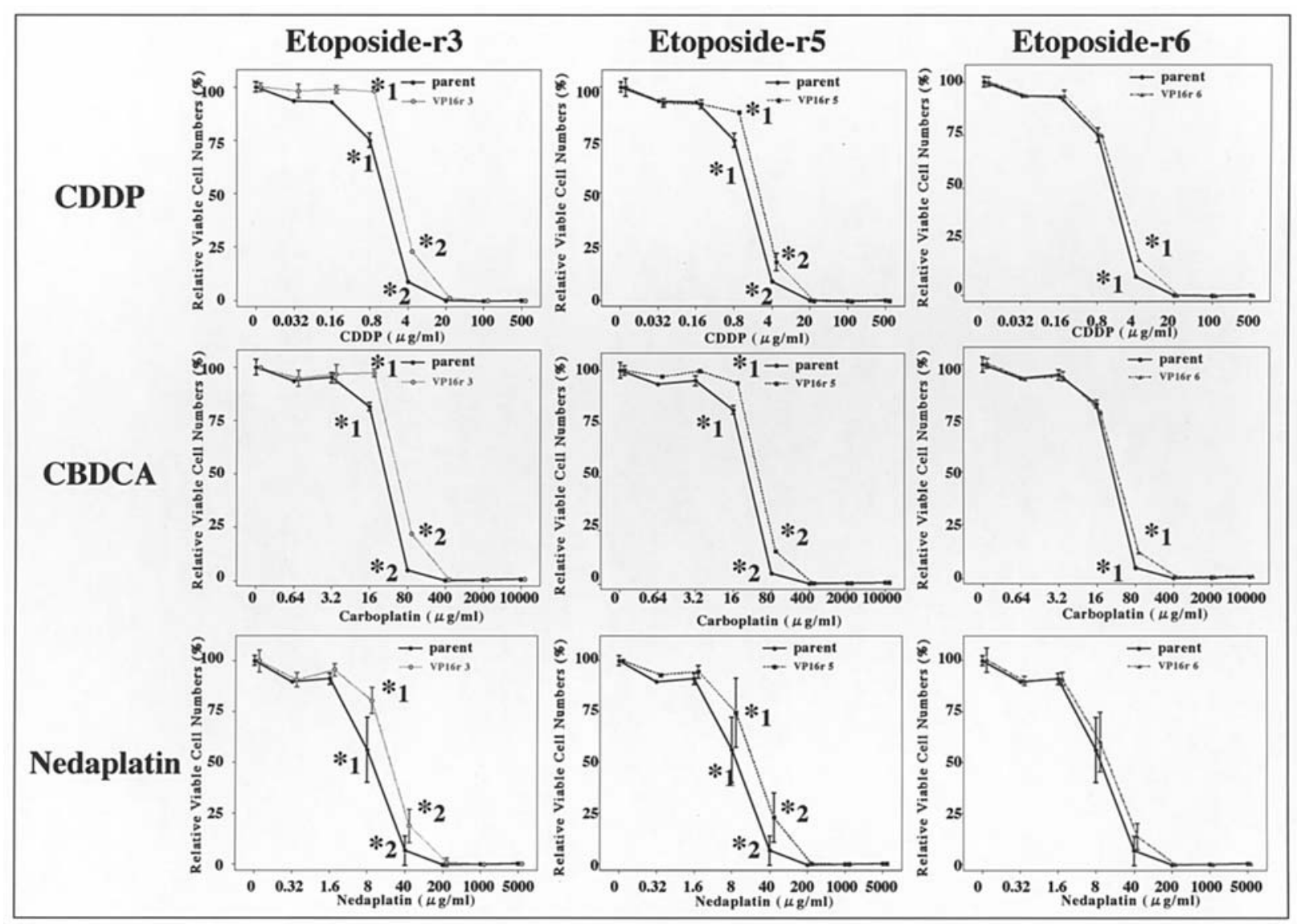

Figure 6. Etoposide-resistant subclones are resistant to platinum anticancer drugs. The anticancer drug sensitivities of the etoposide-resistant subclones to CDDP, CBDCA and nedaplatin were examined. Solid lines with closed circles show the growth-inhibitory curves of the parent ME180 cells. Dotted lines with open circles show the growth-inhibitory curves of the etoposide-resistant subclones. All of the examined etoposide-resistant subclones were significantly more resistant to the anticancer drugs than the parent cells. ${ }^{*} 1-2, \mathrm{p}<0.05$. 


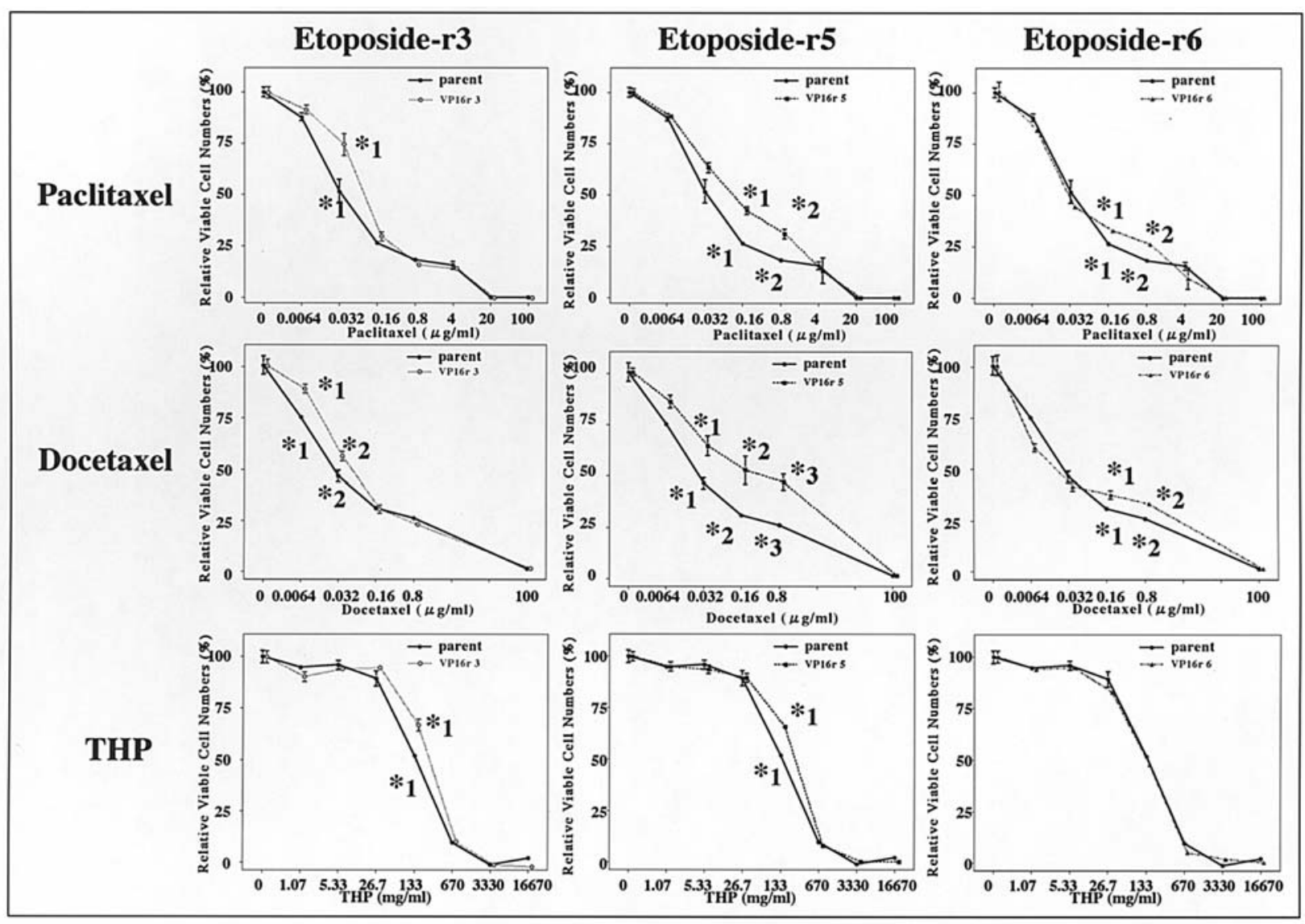

Figure 7. Etoposide-resistant subclones are resistant to paclitaxel, docetaxel and THP. The anticancer drug sensitivities of the etoposide-resistant subclones to taxane compounds (paclitaxel and docetaxel) and a doxorubicin-derivative (THP) were examined. Solid lines with closed circles show the growth-inhibitory curves of the parent ME180 cells. Dotted lines with open circles show the growth-inhibitory curves of the etoposide-resistant subclones. All of the examined etoposide-resistant subclones were significantly more resistant to the anticancer drugs than the parent cells. ${ }^{*} 1-3$, p $<0.05$.

etoposide for 3-5 weeks, and the surviving cells were collected. This collection procedure after etoposide exposure was repeated 4 times. Finally, 6 single cell-derived etoposideresistant subclones, designated Etoposide-r2, Etoposide-r3, Etoposide-r4, Etoposide-r5, Etoposide-r6 and Etoposide-r7, were established by the limiting dilution method (27). The monoclonality of each etoposide-resistant subclone was confirmed by chromosome analysis (data not shown). The establishment of these etoposide-resistant subclones took 12 months.

Cell proliferation and anticancer drug sensitivity assays. Cell proliferation was assayed using the above-mentioned XTT kit. The stimulatory effects of anticancer drugs on cell growth were assayed as follows. Cells in the log phase were detached with $0.25 \%$ trypsin/1 mM EDTA (Gibco-BRL), then cultured overnight in 96 -well plates $\left(5 \times 10^{3}\right.$ cells/well). On day 2, various concentrations of an anticancer drug were added to the cells. On day 4 , the number of viable cells was evaluated using the XTT kit and expressed as the percentage of viable cells $(\%)$ relative to the mean number of viable unstimulated cells. All experiments were performed 3 times to verify the results. The data are shown as the mean $\pm \mathrm{SD}$, and comparative data $(n=6)$ were analyzed by ANOVA.

Flow cytometry. Cells were detached from the culture flask with $3 \mathrm{mM}$ EDTA in phosphate-buffered saline (PBS), and stained according to the following procedure. Cells $\left(3 \times 10^{5}\right)$ were incubated with an excess dose of one of the primary antibodies for $20 \mathrm{~min}$ at $4^{\circ} \mathrm{C}$, then washed twice with washing buffer (PBS containing 2\% FCS and $0.1 \% \quad \mathrm{NaN}_{3}$ ). The cells were then reacted with a secondary antibody, FITC-conjugated goat anti-mouse IgG $(\mathrm{H}+\mathrm{L})$ (Dako Japan, Kyoto) for $20 \mathrm{~min}$ at $4^{\circ} \mathrm{C}$, and washed twice. Finally, the cells were suspended in $200 \mu 1$ of the washing buffer and analyzed with a FACSCalibur ${ }^{\mathrm{TM}}$ (Beckman-Coulter Japan, Tokyo). The primary antibodies were: mouse anti-human CD29 monoclonal antibody (clone TDM29; Cymbus Biotech Ltd., Hampshire, UK), mouse anti-human CD49a monoclonal antibody (clone TS2/7; Serotec Ltd., Oxford, UK), mouse anti-human CD49b monoclonal antibody (clone 31H4; Serotec Ltd.), mouse anti-human CD49c monoclonal antibody (clone 11G5; Cymbus Biotech Ltd.), mouse anti-human CD49d monoclonal antibody (clone 44H6; Cymbus Biotech Ltd.), mouse anti-human CD49e monoclonal antibody (clone SAM1; Beckman-Coulter Japan), mouse anti-human CD49f monoclonal antibody (clone 4F10; Cymbus Biotech Ltd.) and mouse anti-human CD40 monoclonal antibody (clone MAB89; Beckman-Coulter Japan).

\section{Results}

The effects of etoposide during concurrent use with irradiation for cervical SCC were investigated by examining the effects 


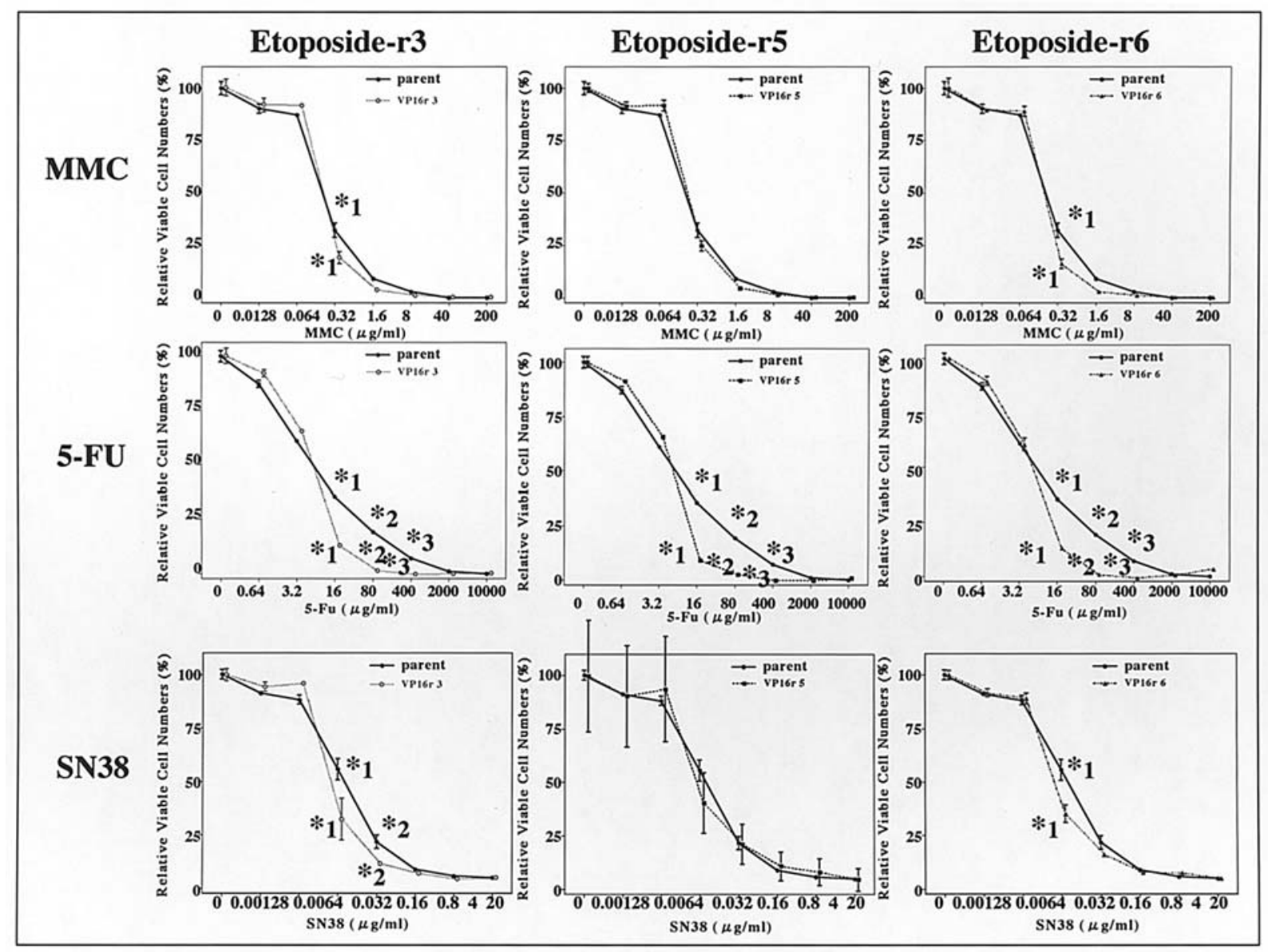

Figure 8. Etoposide-resistant subclones are more sensitive to MMC, 5-FU and SN38 than the parent cells. The anticancer drug sensitivities of the etoposideresistant subclones to MMC, 5-FU and SN38 were examined. Solid lines with closed circles show the growth-inhibitory curves of the parent ME180 cells. Dotted lines with open circles show the growth-inhibitory curves of the etoposide-resistant subclones. All of the examined etoposide-resistant subclones were significantly more sensitive to the anticancer drugs than the parent cells. ${ }^{*} 1-3, \mathrm{p}<0.05$.

of irradiation on the etoposide sensitivity of ME180 cells. As shown in Fig. 1, irradiation significantly reduced the etoposide sensitivity in a dose-dependent manner. A reduction in etoposide sensitivity was found in cells irradiated at $2.5 \mathrm{~Gy}$, indicating that etoposide sensitivity can be reduced with therapeutic doses of irradiation. Since etoposide is usually applied to advanced cervical cancer patients as a drug for tumor dormancy therapy or combination chemotherapy in Japan, we next examined the effects of etoposide as an adjuvant chemotherapy after completion of radiotherapy for unresectable advanced cervical SCC. We established 4 postirradiation-surviving subclones as described in Materials and methods and examined their sensitivities to etoposide. As shown in Fig. 2, 3 of these 4 subclones demonstrated significantly higher etoposide sensitivities than the parent ME180 cells, suggesting that the postirradiation cellular status enhances etoposide sensitivity.

To clarify an optimal chemotherapy protocol with etoposide, we established 6 monoclonal etoposide-resistant subclones as described above. As shown in Fig. 3, the subclones showed significantly reduced etoposide sensitivities, despite retaining a similar microscopic appearance to the parent cells. When their radiosensitivities were examined, 5 of the 6 etoposide-resistant subclones showed radioresistance, while the remaining subclone (Etoposide-r4) was radiosensitive (Fig. 4).
To investigate the mechanisms of the acquired etoposide resistance, the expression of cell adhesion molecules and apoptosis-related receptors was compared among 3 of the etoposide-resistant subclones and parent cells by flow cytometric analyses. As shown in Fig. 5, the 3 etoposideresistant subclones showed apparent reductions in the expression levels of CD29, CD49a and CD49f, which are receptor subunits for extracellular matrices that possibly regulate apoptotic susceptibility $(28,29)$. Notably, the 3 etoposide-resistant subclones revealed significant cell surface expression of CD40, whereas the ME180 parent cells did not.

Finally, drug sensitivity tests were performed on the etoposide-resistant subclones to identify suitable drugs for use in combination with etoposide. As shown in Fig. 6, the etoposide-resistant subclones showed cross-resistance to platinum anticancer drugs, including CDDP, CBDCA and nedaplatin. Since the CD40-CD40L system has been reported to be involved in drug resistance against etoposide, paclitaxel and doxorubicin (18-23), the sensitivities to these kinds of drugs were also examined. As shown in Fig. 7, the 3 etoposideresistant subclones examined also showed cross-resistance to paclitaxel, docetaxel and pirarubicin. However, sensitivities of the etoposide-resistant subclones to MMC, 5-FU and SN38 were significantly higher than those of the parent cells (Fig. 8). The results of all drug sensitivity tests are summarized in Table I. 
Table I. Anticancer drug sensitivities of 3 etoposide-resistant subclones.

\begin{tabular}{lccc}
\hline Anticancer drug & Etoposide-r3 & Etoposide-r5 & Etoposide-r6 \\
\hline Etoposide (VP16) & Resistant & Resistant & Resistant \\
Cisplatin (CDDP) & Resistant & Resistant & Resistant \\
Carboplatin (CBDCA) & Resistant & Resistant & Resistant \\
Nedaplatin & Resistant & Resistant & N.S. \\
Paclitaxel & Resistant & Resistant & Resistant \\
Docetaxel & Resistant & Resistant & Resistant \\
Pirarubicin (THP) & Resistant & Resistant & N.S. \\
Mitomycin C (MMC) & Sensitive & N.S. & Sensitive \\
5-fluorouracil (5-FU) & Sensitive & Sensitive & Sensitive \\
SN38 & Sensitive & N.S. & Sensitive \\
\hline
\end{tabular}

N.S., not significant.

\section{Discussion}

To the best of our knowledge, this is the first study to investigate optimal etoposide chemotherapy protocols for cervical SCC cells. Etoposide has previously been applied to gynecologic malignant tumors as a component drug in combined chemotherapy, and oral etoposide has been used to treat cervical SCC patients in Japan. However, oral etoposide has only been administered to advanced cancer patients as a tumor dormancy therapy. Therefore, we investigated protocols to achieve the most effective antitumor effects of etoposide for treatment of unresectable advanced cervical SCC patients.

Radiotherapy is generally used for patients with unresectable advanced cervical cancer. Although cervical SCC is a well-known radio-sensitive cancer, many studies have reported that concurrent chemoradiotherapy produces better survival ratios than radiotherapy alone (1-8). On the other hand, as described above, chemoradiotherapy may have different therapeutic results depending on the order of treatments (12-16). Therefore, when any combination chemotherapy or chemoradiotherapy is performed, the optimal administration order for each anticancer drug needs to be considered. The present study investigating the effects of etoposide on cervical SCC cells revealed that irradiation dose-dependently reduced etoposide sensitivity, with 3 of 4 postirradiation-surviving subclones showing significantly higher etoposide sensitivity than the parent cells, and 5 of 6 etoposide-resistant subclones showing radioresistance. The reduced etoposide sensitivity after irradiation indicates that concurrent chemoradiotherapy with etoposide may adversely affect the systemic effects of etoposide and produce smaller antitumor effects than the additive effects obtained with irradiation and etoposide. Therefore, etoposide may not be applicable to concurrent chemoradiotherapy. On the other hand, the fact that 3 of the 4 established postirradiationsurviving subclones showed significantly increased etoposide sensitivity indicates that etoposide may be a good anticancer drug for adjuvant chemotherapy after radiotherapy. Most of the established etoposide-resistant subclones had also acquired radioresistance Therefore, etoposide therapy before radiotherapy should not be performed, since radioresistance may be induced by neoadjuvant etoposide therapy.
Optimal chemotherapeutic drugs for combined administration with etoposide would be the anticancer drugs that are more effective on etoposide-resistant subclones than on the parent cells. In the present study, we performed various anticancer drug-sensitivity tests on our etoposide-resistant subclones. The etoposide-resistant subclones showed significant cross-resistance to platinum anticancer drugs, such as CDDP, CBDCA and nedaplatin; taxane compounds, such as paclitaxel and docetaxel; and a doxorubicin derivative, pirarubicin (THP). However, the sensitivities of the etoposideresistant subclones to MMC, 5-FU and SN38 (a major active metabolite of CPT-11) were significantly higher than those of the parent cells. On the other hand, we found that 1 of the 4 postirradiation-surviving subclones showed a lower etoposide sensitivity. Therefore, anticancer drugs such as MMC, 5-FU and CPT-11 should be applied to advanced cervical cancer patients in combination with etoposide, when the latter is used as an adjuvant chemotherapeutic drug after radiotherapy.

One of the most interesting findings of the present study is the increased CD40 expression in etoposide-resistant subclones, which was identified during flow cytometric analyses to elucidate the mechanism of the acquired etoposide resistance. The cell surface expression of receptor subunits for extracellular matrices, such as CD29, CD49a and CD49f, were reduced in the etoposide-resistant subclones. Interestingly, all of the etoposide-resistant subclones showed apparent increases in their cell surface expression of CD40, compared to the parent cells, which showed almost no CD40 expression. These results strongly suggest that the increased CD40 expression has an association with the acquisition of drug resistance and/or radioresistance by the etoposide-resistant cells. CD40 is a cell surface receptor within the TNF receptor family that is expressed on B lymphocytes, dendritic cells, epithelial cells, etc. In B lymphocytes, CD40 ligation rapidly activates the NF- $\mathrm{KB}$ pathway to induce cell proliferation and prevent B-cell antigen receptor-induced cell death (30,31). Several studies have reported that the CD40-CD40L system may be a possible cause of anticancer drug resistance. For example, CD40L inhibited the apoptotic and antiproliferative effects induced by doxorubicin and interfered with caspase-3 activation in non-Hodgkin's lymphoma cell lines (19), while CD40 activation prevented etoposide-induced disruption of 
constitutive $\mathrm{Bax}-\mathrm{Bcl}-\mathrm{X}(\mathrm{L})$ binding to reduce the etoposide sensitivity of a B-cell lymphoma in vitro (21). Furthermore, stimulation of the CD40 receptor inhibited paclitaxel-induced apoptosis in a carcinoma cell line (22), while coexpression and stimulation of CD40 and CD40L on carcinoma cells induced autocrine antiapoptotic signals in cells treated with anticancer drugs (23). However, it is unknown as to whether the CD40-CD40L signals are related to anticancer drugspecific and/or multidrug resistance.

If CD40 signals play a central role in the drug resistance of cancer cells, the prognosis of patients with CD40-positive cancer cells must be poor. Ottaiani et al reported that there was no significant association between CD40 expression and the response to chemotherapy or other clinical and pathological characteristics, and CD40 expression in spindle-cell soft tissue sarcomas was not associated with the response to ifosfamide plus epirubicin chemotherapy (32). This report allows us to propose several possibilities: 1) there is no clinical difference in survival ratio according to the CD40-positivity of cancer cells despite some in vitro relationships among CD40 signals and drug resistance; 2) the relationship between the CD40 expression level and strength of anticancer drug resistance in cancer cells differs among different types of cancers; and 3) no clinical differences in survival ratio can be determined in patients with a soft tissue sarcoma that grows rapidly and causes death within a short period of time. We are currently investigating the possible relationships among clinical therapeutic outcomes and CD40 expression levels in uterine and ovarian cancers.

Our present results indicate that CD40 signals may be a useful molecular target for therapy of incurable patients with multidrug- and/or radio-resistant advanced cervical cancers. We are now searching for key molecules that may be involved in drug resistance and radioresistance.

Flow cytometric studies revealed that cell surface expression of CD40 was induced in our etoposide-resistant subclones. $\mathrm{CD} 40$ has been reported to be a receptor that binds to CD40L and mediates antiapoptotic signaling, in addition to the reported role of the CD40-CD40L system in anticancer drug resistance. As described above, several reports have indicated that the CD40-CD40L system in cancer cells can inhibit the apoptosis induced by various types of anticancer drugs (18-23). In our previous report, CPA-resistant subclones established from the ME180 cell line showed increased CD40 expression, radioresistance and multidrug resistance, including resistance to MMC, 5-FU and SN38 (24). Based on our results for these CPA-resistant subclones, we considered the possibility that increased CD40 expression had a relationship to the acquisition of radioresistance and multidrug resistance. Since the etoposide-resistant subclones established in the present study showed resistance to paclitaxel, etoposide and THP, we cannot exclude the possibility that the CD40-CD40L system is also associated with drug resistance to paclitaxel, etoposide and doxorubicin. Moreover, the present results also indicate that we cannot exclude the possibility of an association between acquired radioresistance and the CD40-CD40L system. However, because the sensitivities of the etoposide-resistant subclones to MMC, 5-FU and SN38 were higher than those of the parent cells, it can at least be concluded that the CD40-CD40L system has no relationship to acquired resistance to these drugs. Therefore, the CD40CD40L system may be restricted to the acquisition of resistance to certain anticancer drugs.

The present results suggest that etoposide should be used as an adjuvant chemotherapeutic drug after the completion of radiotherapy when administered to patients with unresectable advanced cervical SCC. To enhance the effects of etoposide on the cancer cells, it is recommended that MMC, 5-FU and CPT-11 be used in combination chemotherapy with etoposide, since the sensitivities of etoposide-resistant cancer cells to these anticancer drugs can be increased. Regarding the use of oral etoposide therapy, combination chemotherapy with any of the oral 5-FU derivatives may be more effective and easier. We are currently applying novel combination chemotherapies with etoposide plus 5-FU, MMC or CPT-11 to postirradiation patients with advanced cervical SCC after obtaining informed consent.

\section{Acknowledgements}

This study was supported in part by a Grant-in-Aid for Scientific Research from the Ministry of Education, Science, Sports and Culture of Japan and a Grant-in-Aid for Scientific Research from the Ministry of Welfare and Labor of Japan.

\section{References}

1. Morris M, Eifel PJ, Lu J, Grigsby PW, Levenback C, Stevens RE, Rotman M, Gershenson DM and Mutch DG: Pelvic radiation with concurrent chemotherapy compared with pelvic and paraaortic radiation for high-risk cervical cancer. New Eng J Med 340: 1137-1143, 1999.

2. Rose PG, Bundy BN, Watkins EB, Thigpen JT, Deppe G, Maiman MA, Clarke-Pearson DL and Insalaco S: Concurrent cisplatin-based radiotherapy and chemotherapy for locally advanced cervical cancer. New Eng J Med 340: 1144-1153, 1999.

3. Keys HM, Bundy BN, Stehman FB, Muderspach LI, Chafe WE, Suggs CL 3rd, Walker JL and Gersell D: Cisplatin, radiation, and adjuvant hysterectomy compared with radiation and adjuvant hysterectomy for bulky stage IB cervical carcinoma. New Eng J Med 340: 1154-1161, 1999.

4. Whitney CW, Sause W, Bundy BN, Malfetano JH, Hannigan EV, Fowler WC Jr, Clarke-Pearson DL and Liao SY: Randomized comparison of fluorouracil plus cisplatin versus hydroxyurea as an adjunct to radiation therapy in stage IIB-IVA carcinoma of the cervix with negative para-aortic lymph nodes: a Gynecologic Oncology Group and Southeast Oncology Group Study. J Clin Oncol 17: 1339-1348, 1999.

5. Roberts KB, Urdaneta N, Vera R, Vera A, Gutierrez E, Aguilar Y, Ott S, Medina I, Sempere P, Rockwell S, Sartorelli AC, Fischer DB and Fischer JJ: Interim results of a randomized trial of mitomycin $\mathrm{C}$ as an adjunct to radical radiotherapy in the treatment of locally advanced squamous cell carcinoma of the cervix. Int J Cancer 90: 206-223, 2000.

6. Peters WA 3rd, Liu PY, Barrett RJ 2nd, Stock RJ, Monk BJ, Berek JS, Souhami L, Grigsby P, Gordon W Jr and Alberts DS: Concurrent chemotherapy and pelvic radiation therapy compared with pelvic radiation therapy alone as adjuvant therapy after radical surgery in high-risk early-stage cancer of the cervix. J Clin Oncol 18: 1606-1613, 2000.

7. Lorvidhaya V, Chitapanarux I, Sangruchi S, Lertsanguansinchai P, Kongthanarat Y, Tangkaratt S and Visetsiri E: Concurrent mitomycin C, 5-fluorouracil, and radiotherapy in the treatment of locally advanced carcinoma of the cervix: a randomized trial. Int J Rad Oncol Biol Phys 55: 1226-1232, 2003.

8. Eifel PJ, Winter K, Morris M, Levenback C, Grigsby PW, Cooper J, Rotman M, Gershenson D and Mutch DG: Pelvic irradiation with concurrent chemotherapy versus pelvic and para-aortic irradiation for high-risk cervical cancer: an update of Radiation Therapy Oncology Group trial (RTOG) 90-01. J Clin Oncol 22: 872-880, 2004. 
9. Umesaki N, Tanaka T, Miyama M, Morimura M and Ogita S: Postoperative adjuvant chemotherapy with cisplatin, etoposide, and pirarubicin for endometrial carcinoma patients with lymph node metastasis: a pilot study. Oncol Rep 7: 1083-1086, 2000.

10. Umesaki N, Tanaka T, Miyama M and Ogita S: Effect of adjuvant chemotherapy using pirarubicin, cisplatin, and etoposide (PEP) for stage IVB endometrial carcinoma: A case report. Eur J Gynaec Oncol 11: 303-304, 2000.

11. Umesaki N, Izumi R, Fushiki H, Hasegawa K, Kono I, Nishida M, Noguchi H, Okuda H, Sugimori H, Takizawa K, Udagawa Y, Yamamoto K, Tanaka T and Noda K: Cervical adenocarcinoma, a novel combination chemotherapy with mitomycin C, etoposide, and cisplatin for advanced or recurrent disease. Gynecol Oncol 75: 142-144, 1999

12. Tabata T, Takeshima N, Nishida H, Hirai $\mathrm{Y}$ and Hasumi K: A randomized study of primary bleomycin, vincristine, mitomycin and cisplatin (BOMP) chemotherapy followed by radiotherapy versus radiotherapy alone in stage IIIB and IVA squamous cell carcinoma of the cervix. Anticancer Res 23: 2885-2890, 2003.

13. Tanaka $\mathrm{T}$ and Umesaki $\mathrm{N}$ : Radiation reduces pirarubicin sensitivity in human cervical squamous cell carcinoma cells. Oncol Rep 13: 1165-1168, 2005.

14. Tanaka $\mathrm{T}$ and Umesaki N: Effects of mitomycin $\mathrm{C}$ on radiationinduced cell death in human cervical squamous cell carcinomas. Eur J Gynaecol Oncol 26: 411-414, 2005.

15. Tanaka T, Yukawa K and Umesaki N: Irradiation enhances cisplatin sensitivity in human cervical squamous cancer cells in vitro. Eur J Gynaecol Oncol 26: 431-433, 2005.

16. Tanaka T, Yukawa K and Umesaki N: Combination effects of irradiation and irinotecan on cervical squamous cell carcinoma cells in vitro. Oncol Rep 14: 1365-1369, 2005.

17. Clark EA: CD40. In: Cytokine Reference. Oppenheim JJ and Feldmann M (eds). Academic Press, pp1657-1668, 2001.

18. Walker A, Taylor ST, Hickman JA and Dive C: Germinal center-derived signals act with Bcl-2 to decrease apoptosis and increase colonogenicity of drug-treated human B lymphoma cells. Cancer Res 57: 1939-1945, 1997.

19. Voorzanger-Rousselot N, Favrot M and Blay JY: Resistance to cytotoxic chemotherapy induced by CD40 ligand in lymphoma cells. Blood 92: 3381-3387, 1998.

20. Kitada S, Zapata JM, Andreeff M and Reed JC: Bryostatin and CD40 ligand enhance apoptosis resistance and induce expression of cell survival genes in B-cell chronic lymphocytic leukemia. Br J Haematol 1066: 995-1004, 1999.

21. Taylor ST, Hickman JA and Dive C: Epigenetic determinants of resistance to etoposide regulation of $\mathrm{Bcl}-\mathrm{X}(\mathrm{L})$ and $\mathrm{Bax}$ by tumor microenvironmental factors. J Natl Cancer Inst 92: 18-23, 2000.
22. Stumm S, Meyer A, Lindner M, Bastert G, Wallwiener D and Guckel B: Paclitaxel treatment of breast cancer cell lines modulates Fas/Fas ligand expression and induces apoptosis which can be inhibited through the CD40 receptor. Oncology 66: 101-111, 2004.

23. Voorzanger-Rousselot N and Blay JY: Coexpression of CD40 and CD40L on B lymphoma and carcinoma cells. an autocrine anti-apoptotic role. Leuk Lymphoma 45: 1239-1245, 2004

24. Tanaka T, Bai T, Yukawa K and Umeesaki N: Reduced radiosensitivity and increased CD40 expression in cyclophosphamide-resistant subclones established from human cervical squamous cell carcinoma cells. Oncol Rep 14: 941-948, 2005.

25. Lancillotti F, Giandomenico V, Affabris E, Fiorucci G, Romeo G and Rossi GB: Interferon alpha-2b and retinoic acid combined treatment affects proliferation and gene expression of human cervical carcinoma cells. Cancer Res 55: 3158-3164, 1995.

26. Saxena A, Yashar C, Taylor DD and Gercel-Taylor C: Cellular response to chemotherapy and radiation in cervical cancer. Am J Obstet Gynecol 192: 1399-1403, 2005.

27. Lefkovits I: Limiting dilution analysis. In: Immunological Methods. Academic Press, pp355-370, 1979.

28. Chang L, Tanaka T, Umesaki N and Ogita S: Differential apoptotic susceptibility to anti-Fas IgM and anticancer drugs in a human endometrial adenocarcinoma cell line HHUA on laminin and type I collagen. Osaka City Med J 44: 173-180, 1998.

29. Hayashida K, Shimaoka Y, Ochi T and Lipsky PE: Rheumatoid arthritis synovial stromal cells inhibit apoptosis and up-regulate Bcl-XL expression by B cells in a CD49/CD29-CD106-dependent mechanism. J Immunol 164: 1110-1116, 2000.

30. Lalmanach-Girard AC, Chiles TC, Parker DC and Rothstein TL: T cell-dependent induction of NF-kappa B in B cells. J Exp Med 177: 1215-1219, 1993.

31. Schauer SL, Wang Z, Sonenshein GE and Rothstein TL: Maintenance of nuclear factor-kappa $\mathrm{B} / \mathrm{Rel}$ and $\mathrm{c}-\mathrm{myc}$ expression during CD40 ligand rescue of WEHI231 early B cells from receptor mediated apoptosis through modulation of I kappa B proteins. J Immunol 155: 81-86, 1996.

32. Ottaiani A, Pisano C, De Chiara A, Barletta E, Ascierto AP, Botti G, Napolitano M, Apice G and Laffaioli RV: Expression of CD40 has no predictive value in soft tissue sarcomas. Anticancer Res 23: 1597-1604, 2003. 